\title{
Evaluation of Antibacterial and Anti-inflammatory Potential of Withania Somnifera (Ashwagandha) against Salmonella Enterica Serovar Typhimurium \\ Praveen Rishi' ${ }^{1}$, Tanya Sathu ${ }^{1}$, Aman Preet Singh ${ }^{1}$, Simran Preet ${ }^{2}$ \\ Department of Microbiology, Department of Biophysics ${ }^{2}$, Basic Medical Sciences Block, Panjab University, Chandigarh
}

\begin{abstract}
Background \& Objectives: The usual approach to treat Salmonella infections has been the use of conventional antibiotics, but the emergence of MDR (multidrug-resistant) strains and their undesirable effects has diverted the scientific interest towards the use of natural antibacterial and anti-inflammatory compounds. In this context, Withania somnifera (ashwagandha) is one such alternative, which has been safely used for centuries in Indian Ayurvedic medicine for the treatment of various ailments. The present study was therefore, planned to evaluate the antibacterial, anti-inflammatory (edema) and immuno-modulatory potential of the purified whole root extract of Withania somnifera against Salmonella enterica serovar Typhimurium.
\end{abstract}

Material and Methods: Female inbred BALB/c mice, 4-6 weeks old (16-22 gm in weight), were procured from the Central Animal House, Panjab University, Chandigarh (India).

Results: Well-diffusion assay and CFU (colony forming units) enumeration confirmed the in-vitro inhibitory potential of Withania somnifera. The anti-inflammatory potential of ashwagandha was confirmed by mouse paw oedema test and flicking response. Further, a significant decrease in MDA (malondialdehyde) and increase in SOD (superoxide dismutase) levels revealed the modulatory effects of ashwagandha in terms of macrophage functions. Withania somnifera also demonstrated excellent in-vivo potency against serovar Typhimurium as evident by reduction in the number of Salmonellae in the liver, spleen and intestine along with histological studies.

Interpretations \& Conclusion : From the present study, it may be concluded that Withania somnifera possesses strong antibacterial as well as anti-inflammatory potential against Salmonella enterica serovar Typhimurium as evidenced by in-vitro, ex-vivo and in-vivo tests.

Keywords: Ashwagandha, antibacterial activity, ex-vivo, immuno-modulatory, in-vitro, in-vivo, minimum inhibitory concentration.

\section{INTRODUCTION}

Salmonella species cause a variety of infections in humans and domestic animals, ranging from mild food poisoning-gastroenteritis, an acute localized inflammation of the intestine caused by Salmonella

\section{Corresponding Author :}

Dr. Praveen Rishi, Ph.D, FAMI, FABMS

Department of Microbiology

Panjab University, Chandigarh-160014, India

Tel: +91-172-2534146

Email: rishipraveen@yahoo.com

Fax: +91-172-2541770 enterica serovar Typhimurium, to severe systemic lifethreatening illness caused by serovar Typhi and serovar Paratyphi such as enteric fever. During Salmonella infections, the ensuing inflammatory response of the intestinal mucosa is commonly associated with virulence. ${ }^{1,2}$ Infiltrating inflammatory cells participate in the destruction of the invading microbe by the release of certain non enzymatic mediators-oxygen and nitrogen metabolites; enzymatic mediators and cytokines ${ }^{3}$, which attack the polyunsaturated fatty acids in the membrane causing damage to the DNA and proteins. ${ }^{4}$ However uncontrolled release of cytotoxic substances and proinflammatory mediators (Tumor necrosis factor and Interleukins) by the migrating cells resulting into 
oxidative stress, may damage the host tissues as well. Therefore, under such conditions, it is necessary to manage the hyper inflammation level to change the clinical manifestation of the disease. The use of antiinflammatory drugs (antibiotics) is the main strategy for the eradication of this problem, but the increased resistance is an inevitable side effect of the repeated antibiotic use ${ }^{5,6}$, thus increasing the cost of therapy. Studies have shown the increased prevalence of antibiotic resistant Salmonella in humans and animals ${ }^{7-9}$ has resulted into chronic toxicity besides disrupting the normal flora of the host ${ }^{10}$. These shortcomings lead to an urgent global call for natural antibacterial and antiinflammatory compounds which are biocompatible, safer and are cost effective.

Withania somnifera commonly known as ashwagandha has a high repute in the traditional Indian medicine, and is one of the most extensively used plant in Ayurveda and Unani medicine ${ }^{11}$. It is wide spread in Africa, Mediterranean region and the Middle East. ${ }^{12-14}$ It has certain antibacterial, anti-hyperglycemic, anti-oxidant and anti-tumor properties to treat ulcers and senile dementia. Most of its biological activities have been attributed to the presence of certain compounds called withanolides $^{15,16}$. Withania has been demonstrated to possess strong anti-fungal activity and is effective against the treatment of murine aspergillosis ${ }^{17,18}$. Although a lot of work has been carried out on the medicinal applications of Withania somnifera (stem and leaves) $)^{19-21}$, but the antibacterial and immunomodulatory potential of the root extracts of Withania need to be explored more especially against the treatment of severe life threatening bacterial infections. To the best of our knowledge, although there is only one report on its antibacterial activity against Salmonella $a^{22}$, but, the underlying mechanism involved in the modulation currently remains unexplored. Therefore the aim of the present study was to evaluate the strong antibacterial, immuno-modulatory as well as antiinflammatory potential of Withania somnifera against Salmonella enterica serovar Typhimurium as evidenced by in vitro, ex vivo and in vivo studies.

\section{MATERIALSAND METHODS}

\section{Ethics statement}

The experimental protocols were approved by the
Institutional Animal Ethics Committee (Approval ID: IAEC/156 dated 25.08.2011) of Panjab University, Chandigarh, India (Registration number: 45/1999/ CPCSEA) and performed in accordance with the guidelines of Committee for the Purpose of Control and Supervision of Experiments on Animals (CPCSEA), Government of India, on animal experimentation. All the efforts were made to minimize the suffering of animals.

\section{Animals}

Female inbred BALB/c mice, 4-6 weeks old (16-22 gm in weight), were procured from the Central Animal House, Panjab University, Chandigarh (India). Animals were maintained in a well-ventilated room, and were allowed free access to food (Ashirwad Industries Pvt Ltd, Punjab, India) and water ad-libitum.

\section{Bacterial strain and growth conditions}

Standard strain of Salmonella enterica serovar Typhimurium NCTC74, originally procured from Central Research Institute, Kasauli, India, was used in the present study. This strain has been maintained in our laboratory for the last several years and has also been used in recent studies. ${ }^{18,19}$ Stock cultures were prepared and stored at $-80{ }^{\circ} \mathrm{C}$ in glycerol (20\%). Purity of the strain was confirmed biochemically as well as serologically.

For preparation of bacterial cell suspension, bacterial cells grown overnight (at $37{ }^{\circ} \mathrm{C}, 150 \mathrm{rpm}$ ) in nutrient broth $(5.0 \mathrm{gm} / 1$ peptone, $5.0 \mathrm{gm} / 1 \mathrm{NaCl}, 1.5 \mathrm{gm} / 1$ beef extract, $1.5 \mathrm{gm} / \mathrm{l}$ yeast extract, $\mathrm{pH} 7.4 \pm 0.2$ ) were harvested by centrifugation ( $8000 \mathrm{rpm}, 15$ minutes), washed once with $10 \mathrm{mM}$ sodium phosphate-buffered saline (PBS, pH 7.2), and resuspended in PBS to a final concentration of approximately $107 \mathrm{CFU} / \mathrm{ml}$.

\section{Preparation of crude herbal extract}

Commercially available ashwagandha (Withania somnifera) powder, net weight-60gm, manufactured by Dabur, India Ltd., was used for the study. The powder was suspended in $0.5 \%$ carboxymethyl cellulose (CMC, $\mathrm{w} / \mathrm{v}$ in distilled water) serving as a vehicle and was mixed thoroughly using vortex to give final concentrations of $0.5 \%$ to $2 \%$. The powder was stored at room temp in its provided bottle and was kept away from any kind of moisture. 
Screening of antibacterial activity by the agar well diffusion method

The anti-Salmonella activity of Withania somnifera was tested using the modified agar well diffusion method. ${ }^{23}$ Soft nutrient agar plates seeded with $107 \mathrm{CFU} / \mathrm{ml}$ of the Salmonella enterica serovar Typhimurium were prepared. Wells were bored ( $4 \mathrm{~mm}$ diameter) in the agar plates with the help of a sterile cork borer and 100 $\mu 1$ ashwagandha suspension at different concentrations, ranging from $0.5-2 \%$ was dispensed into the wells. The plates were kept at $4^{\circ} \mathrm{C}$ for 1 hour to allow the complete diffusion and finally incubated at $37^{\circ} \mathrm{C}$ overnight. Antibacterial activity was evaluated by measuring the inhibition (clear) zone diameters. Sterile phosphate buffer saline was used as the negative control.

\section{Antibacterial effect of Withania somnifera in terms of colony forming units}

Different flasks containing $20 \mathrm{ml}$ nutrient broth were inoculated with $107 \mathrm{CFU} / \mathrm{ml}$ of Salmonella enterica serovar Typhimurium followed by the addition of different volumes of a single concentration of ashwagandha ( $1 \% \mathrm{w} / \mathrm{v})$. All flasks were incubated under the shaking conditions at $37^{\circ} \mathrm{C}$ overnight. After 24 hours, $100 \mu \mathrm{l}$ of the samples were withdrawn from each flask and spread plated on MacConkey agar plates and observed for enumeration of colony forming units.

\section{Anti-inflammmatory potential of Withania somnifera root extract}

The anti-inflammatory potential of Withania somnifera powdered root extract on Salmonella induced inflammation was assessed by hyperalgesic (flicking) response of the mice inflamed paws. To induce oedema, animals were divided into the four different groups (each comprising of three mice) and were injected with $0.1 \mathrm{ml}$ of the following preparations in the right food pad: (1) $0.1 \mathrm{ml}$ of bacterial suspension $\left(10^{8} \mathrm{CFU} / \mathrm{ml}\right)$, (2) $0.05 \mathrm{ml}$ of bacterial cell suspension $+0.05 \mathrm{ml}$ of ashwagandha ( $1 \% \mathrm{w} / \mathrm{v}$ in $0.5 \% \mathrm{CMC})$, (3) $0.1 \mathrm{ml}$ of $1 \%(\mathrm{w} / \mathrm{v})$ of carrageenan (as positive control) and, (4) $0.1 \mathrm{ml}$ normal saline (as negative control). All the mice were checked at regular intervals of $30 \mathrm{~min}$ for $3 \mathrm{~h}$ for inflammation in the right hind foot pad. After $3 \mathrm{~h}$, hyperalgesic response was assessed by the paw immersion test. Animals were marked on both the hind paws (right and left), just beyond the tibiotarasal junction to ensure the mice paw was dipped to the same level in the water bath at $(47 \pm$ $0.5^{\circ} \mathrm{C}$ ) each time. Paw flicking response in terms of time (sec) in each of the above groups of mice was recorded.

Immuno-modulatory effects of Withania somnifera on macrophage functions (ex-vivo studies)

\section{Extraction of peritoneal macrophages}

Peritoneal macrophages were collected from normal $\mathrm{BALB} / \mathrm{c}$ mice as described by us earlier ${ }^{24}$. Cell viability was checked by $0.2 \%$ trypan blue staining.

Interaction of macrophages infected with Salmonella enterica serovar Typhimurium in the presence and absence of Withania somnifera :

Macrophages (105cells/ml) were infected with Salmonella enterica serovar Typhimurium (107 $\mathrm{CFU} / \mathrm{ml}$ ) at multiplicity of infection 1:100. Extensively washed macrophages were treated with two different concentrations of Withania somnifera i.e. $2 \mathrm{mg} / \mathrm{ml}$ and $4 \mathrm{mg} / \mathrm{ml}$ respectively.

\section{Estimation of lipid peroxidation (LPO):}

Quantitative measurement of lipid peroxidation in the culture supernatants of macrophages was performed according to the method of Wills. ${ }^{25}$ Absorbance of malondialdehyde thus formed was measured at $532 \mathrm{~nm}$. The results were expressed as nanomoles of malondialdehyde (MDA) per milligram of protein, using the molar extinction coefficient of chromophore $\left(1.56 \times 10^{5} \mathrm{M}^{-1} \mathrm{~cm}^{-1}\right)$. The protein content of tissue homogenates was calculated as described previously. ${ }^{26}$

\section{Estimation of superoxide dismutase levels (SOD)}

Levels of antioxidant superoxide dismutase (SOD) in the culture supernatants of macrophages were measured according to the method of Kono. ${ }^{27}$ Change in absorbance was read at $560 \mathrm{~nm}$ for 3 minutes with $30 \mathrm{sec}$ interval. SOD activity was expressed as units of SOD per milligram of protein where one unit of activity is defined as the amount of SOD required to inhibit the rate of reduction of NBT by $50 \%$.

Estimation of antibacterial activity of Withania somnifera against systemic murine salmonellosis (in vivo studies)

Each animal was challenged with a single oral dose of 
Salmonella enterica serovar Typhimurium $(0.1 \mathrm{ml}$ of $107 \mathrm{CFU} / \mathrm{ml}$ ). The Withania somnifera root powder was resuspended in water to get the suspension of the crude drug. The suspension was administered orally, once daily for seven consecutive days with a dose of $100 \mathrm{mg} / \mathrm{kg}$ body weight of the animals, while the control group animals did not receive such treatment and were given normal saline only. On day third, each mouse was challenged with a single dose of Salmonella enterica serovar Typhimurium $(0.1 \mathrm{ml}$ of $108 \mathrm{CFU} / \mathrm{ml})$. Animals in all the challenged groups were sacrificed on 7th day post-Salmonella challenge, whereas all animals in the non-challenged group were sacrificed on 10th day postsupplementation. The assessment of antimicrobial potential of Withania somnifera was demonstrated on the basis of the following parameters:

\section{Bacterial load in the tissue homogenates}

Liver, intestine and spleen of all animals (treated and control mice) were immediately removed after sacrifice, rinsed in isotonic saline solution, and weighed. Ten percent $(\mathrm{w} / \mathrm{v})$ of tissue homogenates were prepared in sterile PBS using a Potter Elvehjem homogenizer. Serial 10-fold dilutions of each homogenate were plated on MacConkey agar medium and Bismuth sulfite agar medium (BSA) for enumeration of CFU per organ after incubation at $37^{\circ} \mathrm{C}$ for 24 hours.

\section{Histopathological studies}

Mice were sacrificed and the intestine, liver and spleen were removed from various groups. These were then fixed in $10 \%$ buffered formalin and processed for histological examination. After fixation in formaldehyde, tissues were dehydrated in different grades of alcohol ie-70\%, 80\%, 90\% and absolute alcohol for 30 minutes, 40 minutes and 1 hour respectively. Tissues were then washed in xylene for 1 hour at room temperature. The washings were repeated using fresh xylene. The tissues were dipped in molten paraffin wax and the wax was quickly cooled to prevent crystallization. Thin sections of tissues were cut with a fine razor attached to Spencer microtome and kept in a water bath at $50^{\circ} \mathrm{C}$ to remove wax. Sections were mounted on separate clean glass microscope slides pretreated for electrostatic adherence. Slides were treated with xylene to remove wax and with alcohol to remove xylene and were then rinsed with water. Slides were further stained in hematoxylin till the time only nuclei get stained and were then rinsed in water. Kept under running water for 2-3 minutes and stained in eosin till sections were bright red and washed in running water till eosin was differentiated. Then slides were blot- dried. After mounting in Distyrene Plasticizer Xylene (DPX), slides were examined under the microscope for histological analysis.

\section{Statistical analysis}

Data were expressed as means \pm standard deviations for three to five independent experiments. Statistical analysis was done by Student's unpaired t test and oneway analysis of variance (ANOVA), followed by pairwise comparison procedures (Tukey test), using Jandel Sigma Stat statistical software, version 2.0. In all cases, statistical significance was defined as having a $\mathrm{P}$ value of $<0.05$.

\section{RESULT \& DISCUSSION}

\section{Antibacterial potential of Withania somnifera (ashwagandha)}

It is clearly indicated from the agar well diffusion assay that ashwagandha, an indigenous medicinal plant, is inhibitory to the growth of Salmonella enterica serovar Typhimurium as evidenced by the zone of growth inhibition in the range of 3-18 $\mathrm{mm}$ diameter around the wells filled with $100 \mu \mathrm{l}$ of different concentrations of ashwagandha used while no zone of inhibition was observed around the control wells (Figure $1 \mathrm{a} \& \mathrm{~b}$ ). This may be attributed to the presence of important

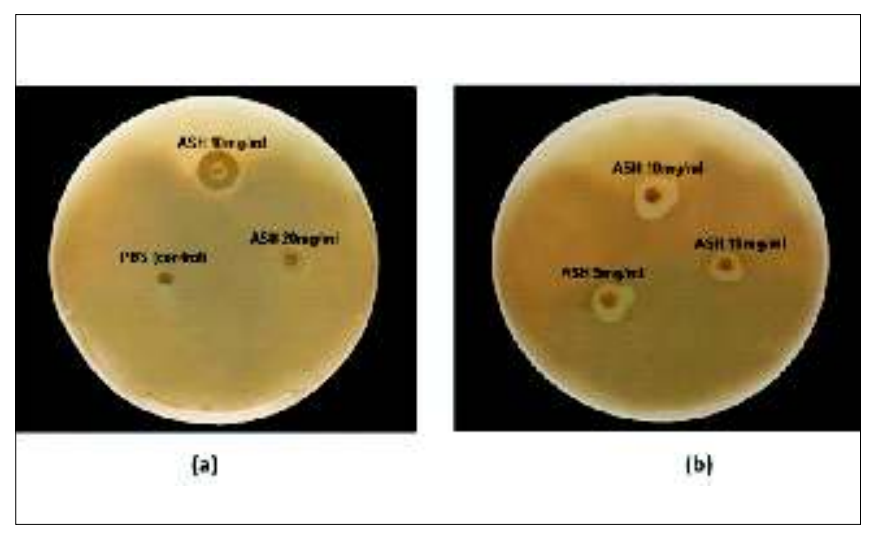

Fig. I. Radial well diffusion assay showing zone of inhibition produced by $100 \mu \mathrm{l}$ of different concentrations of ashwagandha against Salmonella enterica serovar Typhimurium 
Table I

Log10 CFU/ml of Salmonella in the presence of Withania somnifera $(100 \mu \mathrm{l}$ of $10 \mathrm{mg} / \mathrm{ml})$

\begin{tabular}{|ll|}
\hline Groups & $\begin{array}{l}\text { Log10 CFU/ml of Salmonella } \\
\text { enterica serovar Typhimurium } \\
\text { after 24 hr incubation } \\
\text { (mean } \pm \text { S.D.) }\end{array}$ \\
\hline $\begin{array}{l}\text { Growth of Salmonella enterica } \\
\text { serovar Typhimurium in the } \\
\text { absence of Withania } \\
\text { somnifera (control) }\end{array}$ \\
\hline $\begin{array}{l}\text { Growth of Salmonella enterica } \\
\text { serovar Typhimurium in the } \\
\text { presence of Withania } \\
\text { somnifera (test) }\end{array}$ \\
\hline
\end{tabular}

Values are expressed as mean \pm S.D. of three individual observations.

*Significant difference $(\mathrm{p}<0.05)$ from control group.

antibacterial compounds like withanolides and withaferin present in the roots of Withania somnifera.

\section{Antibacterial effect of Withania somnifera in terms of colony forming units}

The bactericidal effect of the purified root extract of Withania somnifera was also evaluated against Salmonella enterica serovar Typhimurium in terms of colony forming units (CFU). After 24 hours of incubation with ashwagandha preparation, a log unit decrease of 4.18 was observed in the colony forming units of Salmonella, thus confirming the anti-Salmonella

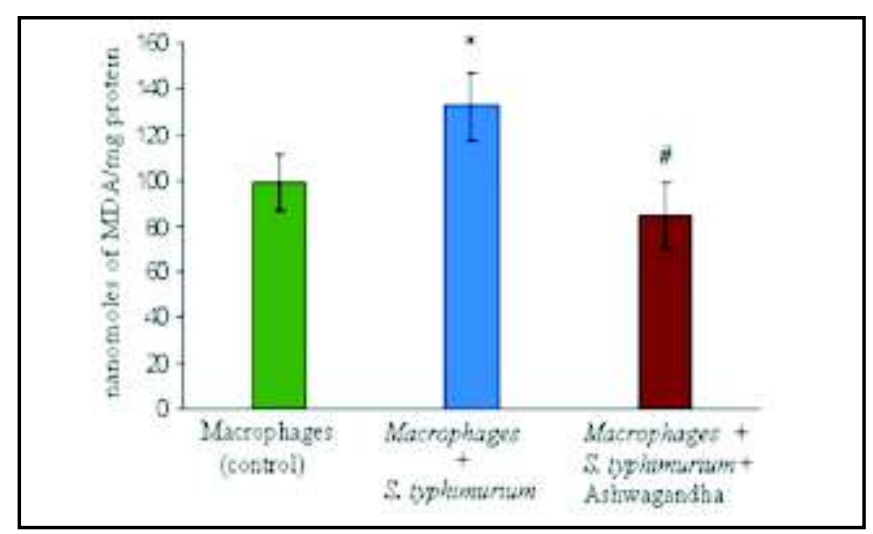

a activity of ashwagandha (Table I).

\section{Measurement of malondialdehyde (MDA) levels}

Significantly increased MDA level was observed in the supernatant of macrophages infected with Salmonella enterica serovar Typhimurium (132.36 \pm 14.60 nanomoles/mg protein, $\mathrm{p}<0.05)$ as compared to that of uninfected macrophages (control, $99.46 \pm 11.90$ nanomoles/mg protein) (Figure 2a). However, when the macrophages were infected with Salmonella enterica serovar Typhimurium in the presence of ashwagandha, a significant decrease in the MDA level was observed $(84.61 \pm 15.03$ nanomoles/mg protein, $\mathrm{p}<0.05)$. Therefore, it may be suggested that ashwagandha has the potential to scavenge the free radicals thereby decreasing the extent of cell damage. It has been shown that treatment with the extracts of Withania somnifera significantly reduces the tissue injury leading to the decreased peroxidation. ${ }^{28}$

\section{Measurement of SOD levels}

Significant decrease in the SOD activity $(\mathrm{p}<0.01)$ was observed when macrophages were infected with Salmonella (7.34 \pm 1.36 units/mg protein) as compared to that of uninfected macrophages (12.46 \pm 2.14 units/mg protein). SOD activity might have been decreased due to the consumption of antioxidants to scavenge the oxidants or free radicals. In addition, the efficacy of the antioxidant defense system may be impaired during inflammation, partly as a result of autooxidation. ${ }^{29}$ However, treatment of infected

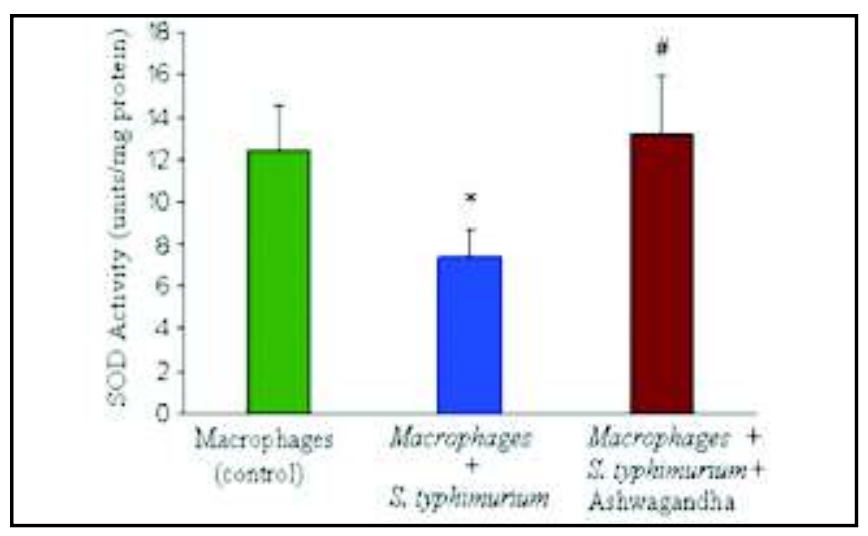

b

Fig. 2. (a) Effect of ashwagandha on the MDA levels of macrophages infected with S. enterica serovar Typhimurium. Values are expressed as mean $\pm S D$ of three individual observations. "Shows significant difference ("p<0.05) from uninfected macrophages (control): \# Shows significant difference $(* p<0.05)$ from uninfected macrophages infected with Salmonella enterica serovar Typhinurium. (b) Estimation of SOD activity of macrophages infected with + S.D. of three individual observations. "Shows significant difference ( $\left.{ }^{*}<0.01\right)$ from uninfected macrophages (control). " Shows significant difference $\left({ }^{*} p<0.01\right)$ from macrophages infected with Salmonella enterica serovar Typhimurium. 
macrophages with the ashwagandha resulted in a significant increase in the SOD activity $(13.24 \pm 2.7$ units $/ m g$ protein, $\mathrm{p}<0.01$ ) (Figure $2 \mathrm{~b}$ ). It has been shown that active components of Withania like sitoindosides and withaferin-A (glycowithanolides), significantly increased the antioxidant activities of SOD, catalytically scavenging superoxide radicals and thus helps to protect the host tissues from oxidative damage. ${ }^{30}$

\section{Anti-inflammatory potential of Withania somnifera (ashwagandha)}

Paw flicking response depicts the flicking response of mice with inflamed paw, indicating thermal hyperalgesia (Figure 3). The time required for the withdrawal of the paw injected with Salmonella enterica serovar Typhimurium cell suspension was significantly

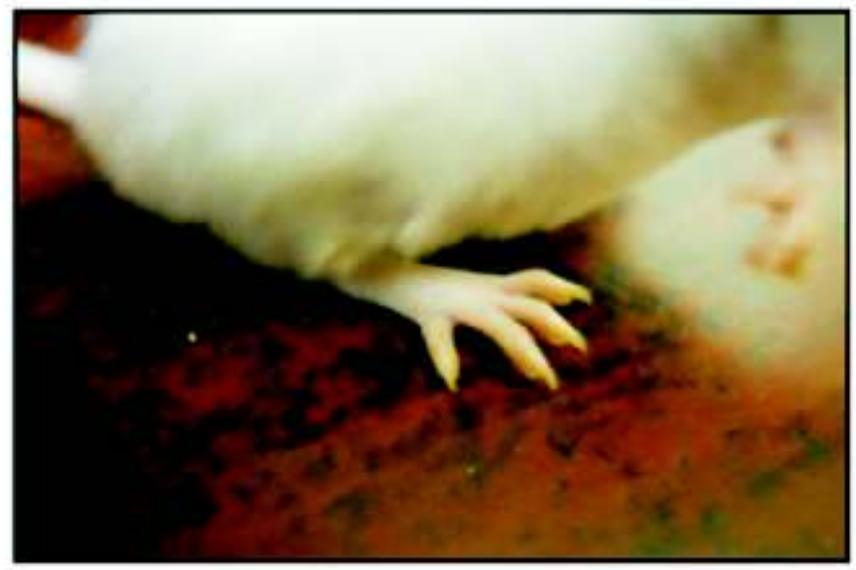

(a)

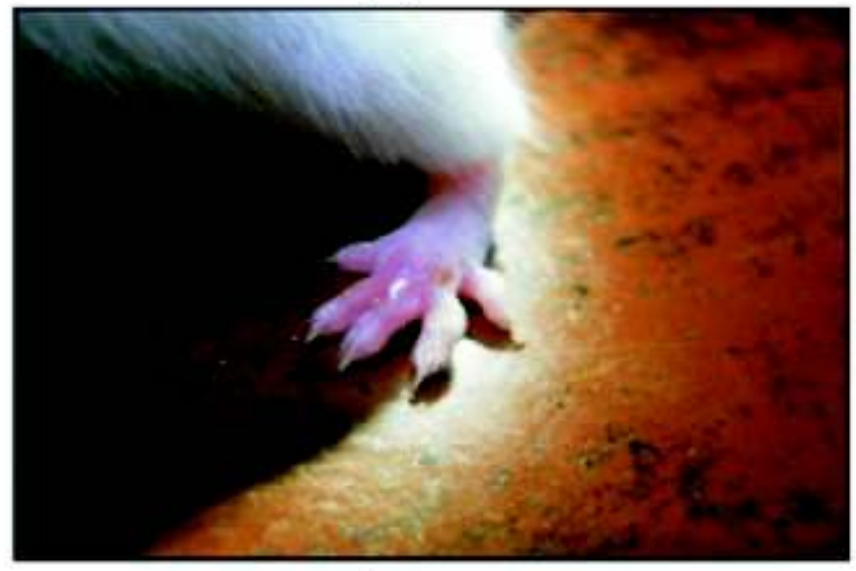

(c)

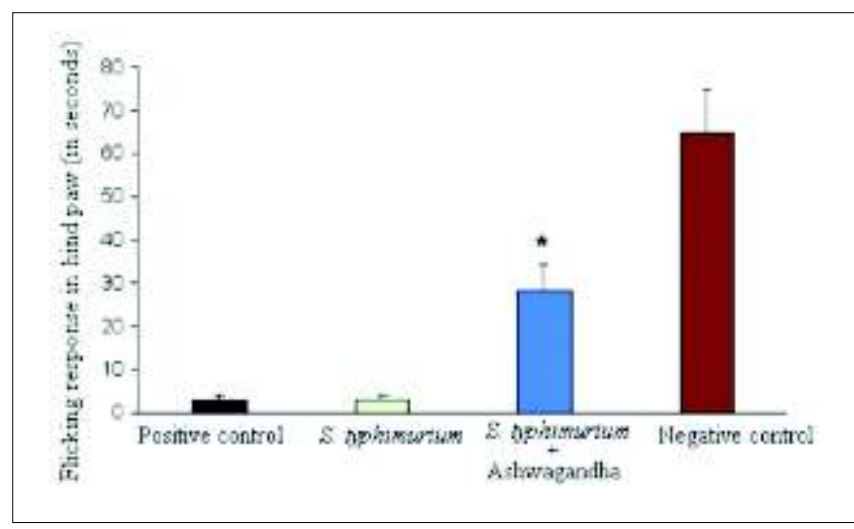

Fig. 3. Flicking response observed after 3 hours in mice hind paw injected with Salmonella enterica serovar Typhimurium in the absence and presence of ashwagandha. Values are expressed as mean \pm S.D. of three individual observations. *Shows significant difference $(* p<0.01$ from serovar Typhimurium -infected group.

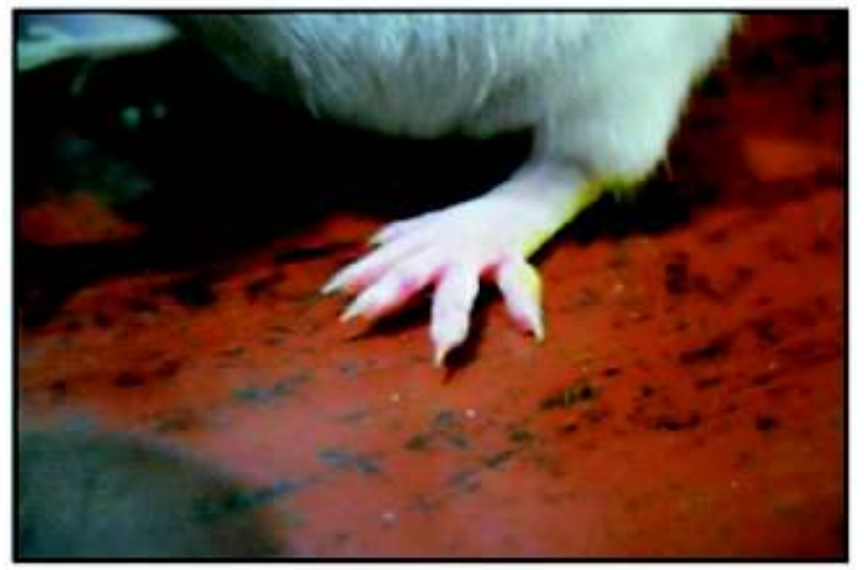

(b)

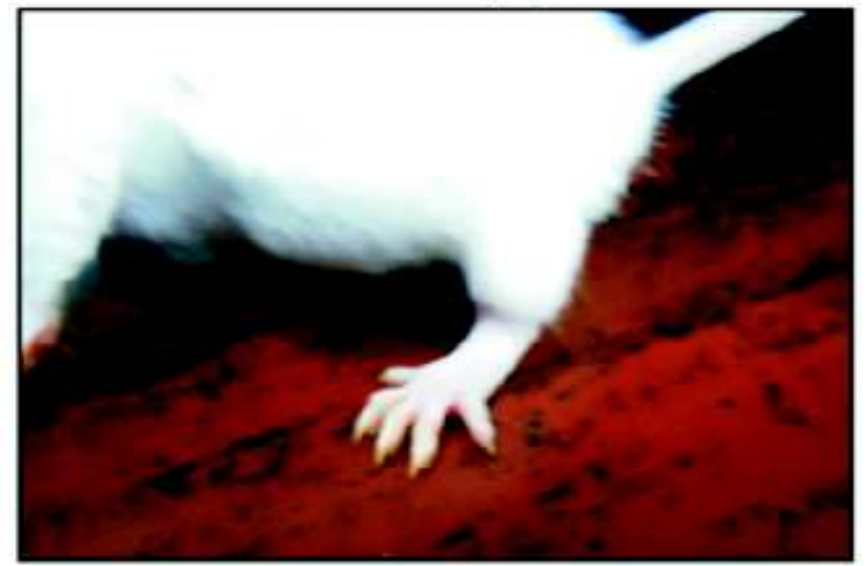

(d)

Fig. 4. (a) Mouse injected with normal saline on the dorsal foot pad of right paw indicating no signs of inflammation (b) MouseRadial well diffusion assay showing zone of inhibition produced by $100 \mu$ l of different concentrations of ashwagandha against Salmonella enterica serovar Typhimurium (c) Mouse showing inflammation in the left paw injected with carrageenan (positive control) (d) Mouse showing inflammation in the dorsal foot pad of the left paw injected with Salmonella enterica serovar Typhimurium and ashwagandha extract. 
shorter $(\mathrm{p}<0.001)$ than that of the control paw injected with normal saline, indicating hyperalgesia. Thermal hyperalgesic response significantly reduced upon treatment with the root extract of Withania somnifera (ashwagandha) as the time required for the withdrawal of mice paw injected with serovar Typhimurium cell suspension in the presence of ashwagandha was significantly longer when compared to the infected group $(\mathrm{p}<0.001)$, indicating analgesic effect of ashwagandha. The observed anti-inflammatory activity of ashwagandha might be due to the presence of withanolides as the main component of the extract, giving relief from pain induced by inflammation.

\section{Qualitative assessment of inflammation (paw oedema test)}

No inflammation was observed in the hind paw of mice injected with normal saline shown in (Figure 4a) which served as negative control. Inflammation was found to be maximum in the mice injected with Salmonella (Figure 4b) which was at par with that induced by carrageenan (positive control, Figure 4c). However, ashwagandha preparation significantly reduced oedema induced by Salmonella enterica serovar Typhimurium cell suspension (Figure 4d).

\section{Bacterial load in liver, intestine and spleen}

Supplementation with Withania somnifera significantly $(p<0.05)$ decreased the bacterial load in the tissue homogenates of the challenged groups (Figure 5). The

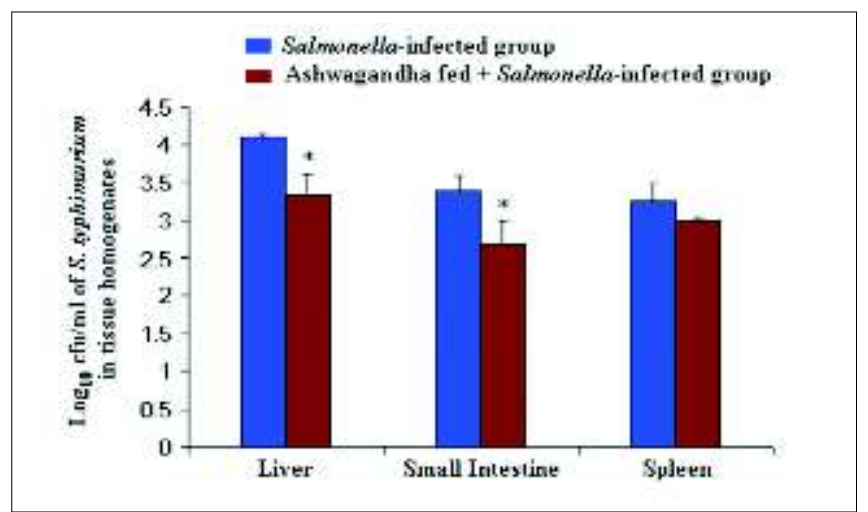

Fig. 5. Bacterial load in the liver, small intestine and spleen of mice challenged with Salmonella. Values are expressed as mean + S.D. of three individiual observations. *Shows significant difference $(* p<0.05)$ from Salmonella infected group.

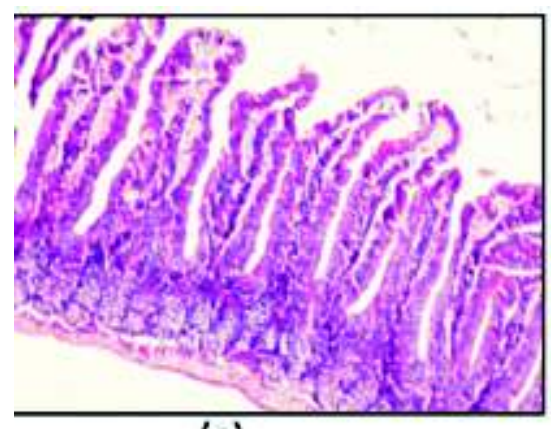

(a)

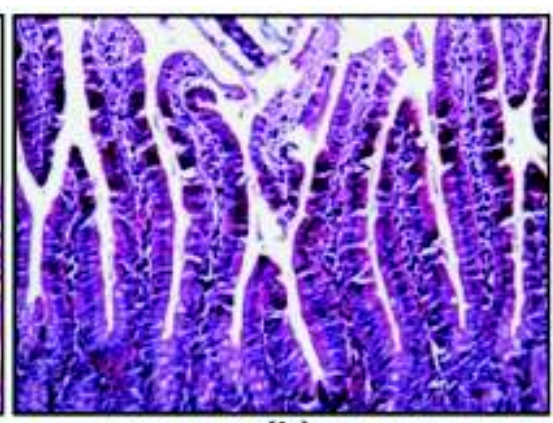

(b)

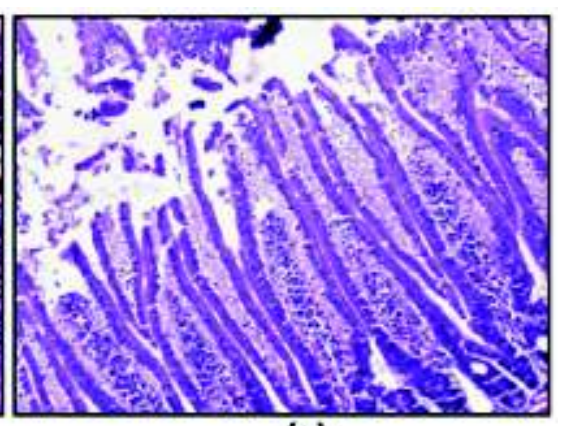

(c)

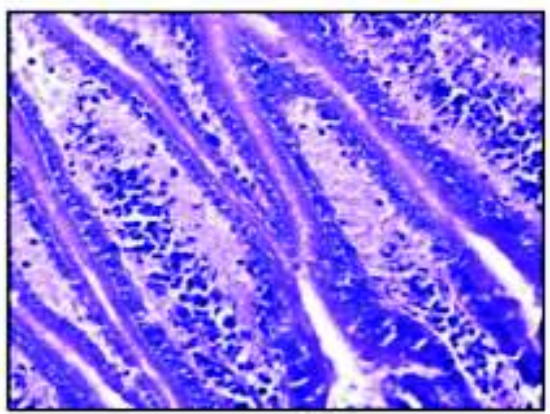

(d)

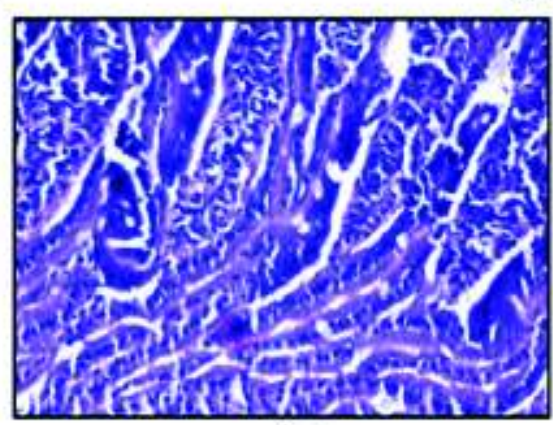

(e)

Fig. 6 : Photomicrograph of mince small intestine section (a) Photomicrograph of the normal / control mouse showing intact crypts and villi (100X); (b) Photomicrograph of ashwagandha per se mouse showing intact crypts and villi (100 X); (c) Photomicrographs of Salmonella infected mouse showing damaged villi and crypts, ileum is swollen and is inflamed. Lamina limitence is expanded by lymphocytic infiltration (severe ileitis) $(100 \mathrm{X})$.(d) Photomicrograph of mouse after Salmonella infection (400 X); (e) Photomicrograph of mouse supplemented with ashwagandha before Salmonella challenge showing decreased inflammation and less expansion of lamina limitence (mild ileitis) (400 X). H and Estain. 
results of the present study clearly demonstrate that such treatment successfully obliterated the severity of infection as evident from the reduction in bacterial load in different vital organs. This activity of Withania somnifera may be attributed to the presence of strong immuno-potentiating compounds present in the root extract. ${ }^{31,32}$ In fact, the compounds present in the root extract might be successful in activation of the immune components of the host, leading to the observed increase in phagocytosis and intracellular killing by peritoneal macrophages. Therefore, the possibility of macrophage activation by Withania somnifera treatment in conjunction with antibacterial property of the active constituents responsible for eliminating the pathogens may be speculated.

\section{Histological studies}

Histological evaluation of small intestine sections did not reveal any morphological alterations in the control
(Figure 6a) and ashwagandha per se group (Figure 6b). Small intestine from Salmonella enterica serovar Typhimurium infected mice showed damaged villi and crypts (Figure $6 \mathrm{c}$ ). There was heavy amount of inflammation and foam cells and lymphocytic infiltration (Figure $6 \mathrm{~d}$ ). Lamina limitence was observed to be expanded by infiltration of lymphocytes (severe ileitis). However, histological analysis of the small intestine sections from mice supplemented with ashwagandha prior to Salmonella challenge revealed decreased inflammation of lymphocytes and foam cells as compared to the infected group (mild ileitis) (Figure 6 e). Histological evaluation of liver tissues did not reveal any morphological alterations in the control (Figure 7a) and ashwagandha per se group (Figure 7b). In contrast, liver sections from Salmonella enterica serovar Typhimurium infected group revealed marked histological alterations such as damaged central vein, lymphocytic infiltration with focal area of necrosis and

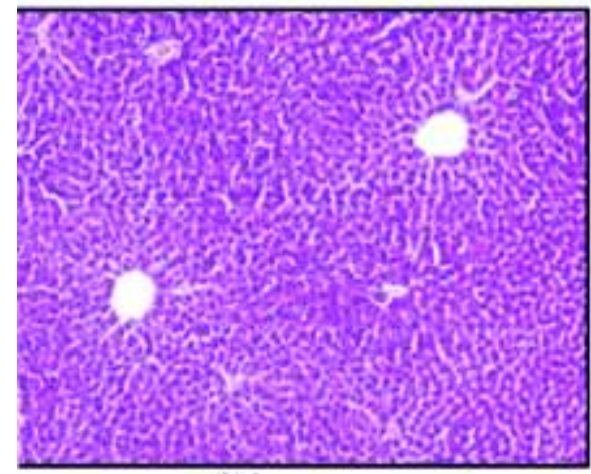

(a)

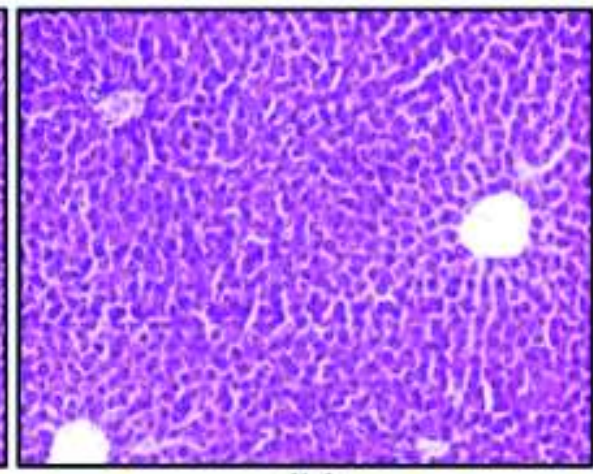

(b)

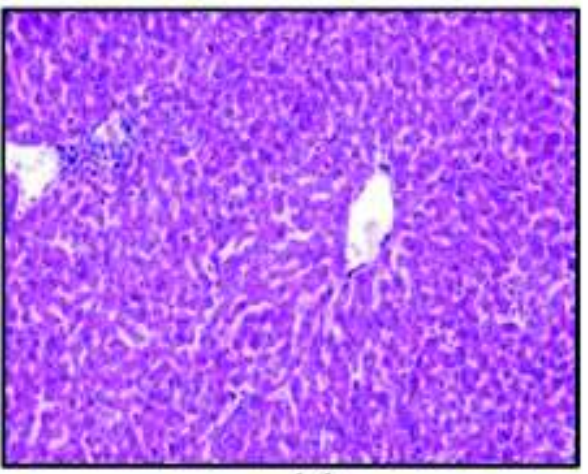

(c)

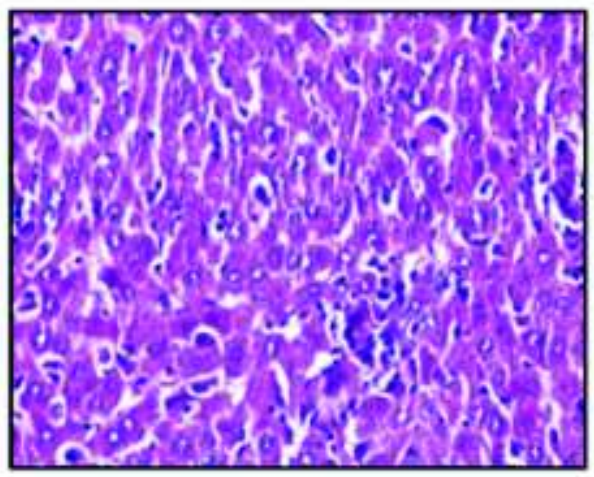

(d)

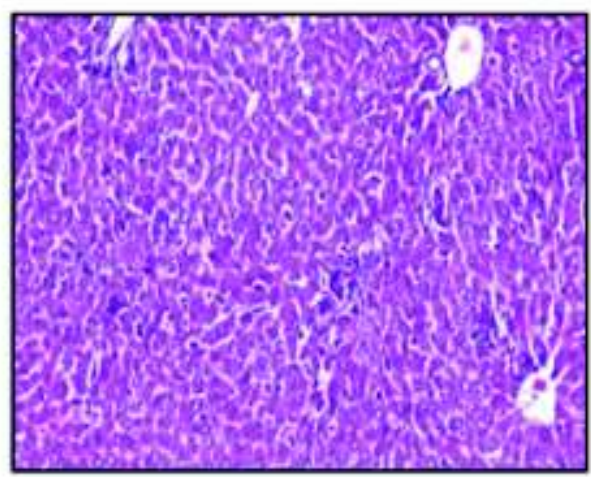

(e)

Fig. 7. Photomicrograph of mice liver section (a) Photomicrograph of the normal / control mouse liver showing normal morphology (200 X); (b) Photomicrograph of liver section from mouse supplemented with ashwagandha (per se) for ten days showing normal liver morphology (200 $X$; (c) Photomicrographs of liver section from Salmonella-challenged mouse showing disrupted central vein, lymphocytic infiltration with focal area of necrosis and heavy Kupffer cell hyperplasia $(200 \mathrm{X})$; (d) Photomicrographs of liver section from Salmonella-challenged mouse supplemented with ashwagandha showing normal liver lobules with mild inflammation and mild Kupffer cell hyperplasia (200X). H and E stain. 
Kupffer cell hyperplasia (Figure 7 c \& Figure 7d). Supplementation with ashwagandha (Figure VII e) resulted in significant morphological protection in terms of marked reduction in inflammation and hepatocyte damage in Salmonella-infected mice.

From the present study, it may be concluded that Withania somnifera possesses strong antibacterial as well as anti-inflammatory potential against Salmonella enterica serovar Typhimurium as evidenced by in-vitro, ex-vivo and in-vivo tests. Interestingly, the in-vivo studies suggesting the oral administration of aqueous root extracts of this plant successfully obliterated Salmonella infection in Balb/C mice. However, further evaluation of the protective efficacy against clinical isolates of Salmonella could be the next key step to confirm the value added potential of this herb. Finally, this study can serve as the pioneer study to evaluate the strategy of using Withania somnifera in conjunction with other herbs or drugs having direct effect on the pathogen.

\section{Conflicts of interest: None.}

\section{REFERENCES}

1. Giannella RA, Gots RE, Charney AN, Greenough WB, Formal SB. Pathogenesis of Salmonella-mediated intestinal fluid secretion. Gastroenterol 1975;69:1238-45.

2. Giannella RA. Importance of the intestinal inflammatory reaction in Salmonella-mediated intestinal secretion. Infect Immun 1979;23:140-5.

3. Henson R, Johnston T. Virulence associated with inflammatory response in case of Salmonella infection. J Microbiol 1987;24:23-32.

4. Ballmer G, Marshall PA, Olsen SJ, Villar RG. Effect of Reactive oxygen species or radicals on the generation of PUFA's in the damage and introduction about the DNA damage. Ind J Exp Biol 1994;35:345-56.

5. Threlfall EJ, Frost JA, Ward LR, Rowe B. Increasing spectrum of resistance in multi-resistant Salmonella enterica serovar Typhimurium. Lancet 1996;347: 1053-4.

6. Rishi P, Preet S, Kaur P. Effect of L. plantarum cell free extract and cotrimoxazole against Salmonella enterica serovar Typhimurium: a possible adjunct therapy. Ann Clin Microbial Antimicrob 201;10:9-17.

7. Angulo FJ, Johnson KR, Tauxe RV, Cohen ML. Origins and consequences of antimicrobial resistant non typhoidal Salmonella, implications for the use of flouroquinolones in food animals. Microb Drug Resist 2000;6:77-83.

8. Gross U, Tschape H, Bednarek I, Frosch M. Antibiotic resistance in Salmonella enterica serotype Typhimurium. Eur J Clin Microbiol Infect Dis 1998;17:385-7.

9. O'Brien TF. Emergence, spread, and environmental effect of antimicrobial resistance: how use of an antimicrobial anywhere can increase resistance to any antimicrobial anywhere else. Clin Infect Dis 2002;34:S78-S84.

10. Dundas Y, Heffron G, Kyumer U, Serent R. Studies on the toxicity effects due to the use of antibiotics and disruption of the normal flora of the host. Eur J Med Microbiol 1999; 23:345-56.

11. Chopra RN, Chopra IC, Handa KL, Kapur LD. Indigenous drugs of India. UN Dhar and Sons, Calcutta, India, 1958.

12. Mirjalili MH, Moyano E, Bonfill MR, Cusido M, Palazon J. Steroidal Lactones from Withania somnifera, an ancient plant for novel medicine. Molecules 2009;14: 2373-93.

13. Mabberley DJ. The Plant Book, 2nd edn. Cambridge University Press, Cambridge, 1997.

14. Doaigey AR. Occurrence, type and location of calcium oxalate crystals in leaves and stem of 16 species of poisonous plants. Am J Bot 1991;78:1608-16.

15. Khanna PK, Kumar A, Ahuja A, Kaul MK. Biochemical composition of roots of Withania somnifera (L.) dunal. Asian J Plant Sci 2006;5:1061-3.

16. Takeuchi K, Tomita H, Fujimoto S, Kudo M, Kuwano H, Ike Y. Antibacterial activity of plant extracts and phytochemicals. FEMS Microbiol Lett 2005;243:347-54.

17. Dhuley JN. Therapeutic efficacy of ashwagandha against experimental aspergillosis in mice. Immunopharmacol Immunotoxicol 1998;20:191-8

18. Ziauddin P, Phansalkar N, Pataki M, Diwanay PS, Patwardhan B. Studies on the immuno-modulatory effects of ashwagandha. J Ethnopharmacol 1996;50:69-76.

19. Elsakka M, Grigorescu EU, Stanescu U, Dorneanu V. New data referring to chemistry of Withania somnifera species. Rev Med Chir Soc Med Nat Iasi 1990; 94:385-7.

20. Arora S, Dhillon S, Rani G, Nagpal A. The in vitro antibacterial synergistic activities of Withania somnifera extracts. Fitoterapia 2004; $75: 385-8$

21. Sundaram S, Dwivedi P, Purwar S. In vitro evaluation of antibacterial activities of crude extracts of Withania somnifera (Ashwagandha) to bacterial pathogens. Asian J Biotechnol 2011;3(2):194-9.

22. Owais M, Sharad KS, Shehbaz A, Saleemuddin M. Antibacterial efficacy of Withania somnifera (ashwagandha) an indigenous medicinal plant against experimental murine salmonellosis. Phytomedicine 2005; 12:229-35.

23. Sarkar PK, Banerjee S. Antibacterial activity of the bacterial isolates obtained from natural habitats. J Food Sci Technol 1996;33:231-3. 
24. Chander H, Majumdar S, Sapru S, Rishi P. Macrophage cell death due to Salmonella enterica serovar Typhi and its role in apoptosis. Microbiol Immunol 2005;49:323-30.

25. Wills ED. Mechanism of lipid peroxidation formation in animal tissues. J Biochem 1966;99:667-76.

26. Lowry OH, Rosbrough NJ, Randal RJ. Protein estimation with Follin's reagent. J Biol Chem 1951;193:265-75.

27. Kono Y. Generation of superoxide radical during autooxidation of hydroxylamine and an assay of superoxide diamutase. Arch Biochem Biophy 1978;186:189-95.

28. Palazon R. In vivo growth inhibitory effect of Withania somnifera (ashwagandha) on a transplatable mouse tumor, sarcoma. Ind J Exp Biol 2009;45:67-90.
29. Nieto J, Cleveland KL, Vieira S, Caleja C. Efficacy of antioxidant defense mechanism during inflammation. Infect Immun 2000;54:554-9.

30. Sankar H, Manivasagam D. Effect of higher doses of ashwagandha on superoxide levels in the cultured mouse macrophages. J Nat Med 2007;67:436-78.

31. Agarwal R, Diwanay S, Patki P, Patwardhan B. Studies on immuno-modulatory activity of Withania somnifera (ashwagandha) extracts in experimental immune inflammation. JEthanopharmocol 1999;67:27-35.

32. Mishra LC, Singh BB, Dagenais S. Scientific basis for the therapeutic use of Withania somnifera (ashwagandha): a review. Altern Med Rev 2000;5:334-46. 